\title{
Next-generation sequencing to guide cancer therapy
}

\author{
Jeffrey Gagan ${ }^{1}$ and Eliezer M. Van Allen $2,3^{*}$
}

\begin{abstract}
As a result of multiple technological and practical advances, high-throughput sequencing, known more commonly as "next-generation" sequencing (NGS), can now be incorporated into standard clinical practice. Whereas early protocols relied on samples that were harvested outside of typical clinical pathology workflows, standard formalin-fixed, paraffin-embedded specimens can more regularly be used as starting materials for NGS. Furthermore, protocols for the analysis and interpretation of NGS data, as well as knowledge bases, are being amassed, allowing clinicians to act more easily on genomic information at the point of care for patients. In parallel, new therapies that target somatically mutated genes identified through clinical NGS are gaining US Food and Drug Administration (FDA) approval, and novel clinical trial designs are emerging in which genetic identifiers are given equal weight to histology. For clinical oncology providers, understanding the potential and the limitations of DNA sequencing will be crucial for providing genomically driven care in this era of precision medicine.
\end{abstract}

\section{Introduction}

Many biological discoveries about cancer have been the product of a reductionist approach, which focuses on modeling phenomena with as few major actors and interactions as possible $[1,2]$. This reductionist thinking led the initial theories on carcinogenesis to be centered on how many "hits" or genetic mutations were necessary for a tumor to develop. It was assumed that each type of cancer would progress through a similar, if not identical, process of genetic hits. Indeed, there are a handful of

\footnotetext{
* Correspondence: eliezerm_vanallen@dfci.harvard.edu

${ }^{2}$ Department of Medical Oncology, Dana-Farber Cancer Institute, Boston, MA 02115, USA

${ }^{3}$ Broad Institute of MIT and Harvard, Cambridge, MA 02142, USA

Full list of author information is available at the end of the article
}

cancer types, such as chronic myelogenous leukemia, that feature a single and pathognomonic DNA mutation. Working on this assumption, early methods to explore the genomic foundations of different cancers involved targeted exploration of specific variants and genes in a low-throughput fashion [3]. However, most cancers are genetically complex, and are better defined by the activation of signaling pathways rather than a defined set of mutations. The success of the Human Genome Project inspired similar projects looking at the genome in various cancers [4]. That success, along with the increased affordability and reliability of sequencing [5], has led to the integration of genome science into clinical practice. The use of these data to assist in diagnosis is generally referred to as precision medicine [6, 7].

Next-generation sequencing (NGS), also known as massively parallel sequencing, represents an effective way to capture a large amount of genomic information about a cancer. Most NGS technologies revolve around sequencing by synthesis [5]. Each DNA fragment to be sequenced is bound to an array, and then DNA polymerase adds labeled nucleotides sequentially. A high-resolution camera captures the signal from each nucleotide becoming integrated and notes the spatial coordinates and time. The sequence at each spot can then be inferred by a computer program to generate a contiguous DNA sequence, referred to as a read.

Multiple technological enhancements have allowed NGS to be more readily implemented in a clinical workflow (Fig. 1). Samples now no longer need to be handled differently from standard diagnostic specimens, and recent advances have even enabled increasingly complex genomic data to be derived from a patient's peripheral blood. The concept of precision medicine goes hand in hand with an understanding of the cancer genome as determined by NGS. In this review, we will explore the expanding NGS methodologies, analytical methods, and clinical applications that are driving precision cancer medicine.
Ciomed Central

C 2015 Gagan and Van Allen. This is an Open Access article distributed under the terms of the Creative Commons Attribution License (http://creativecommons.org/licenses/by/4.0), which permits unrestricted use, distribution, and reproduction in any medium, provided the original work is properly credited. The Creative Commons Public Domain Dedication waiver (http:// creativecommons.org/publicdomain/zero/1.0/) applies to the data made available in this article, unless otherwise stated. 


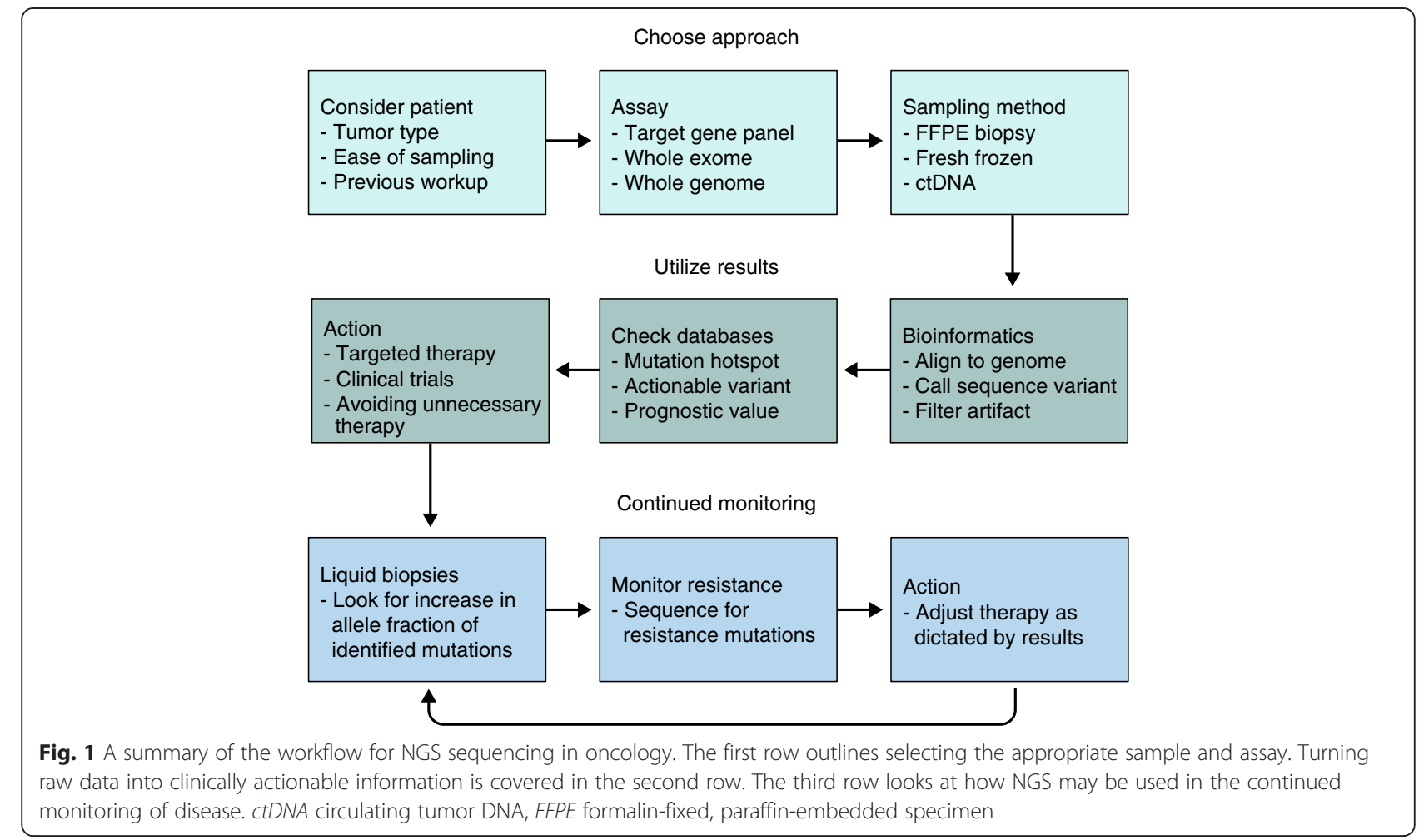

\section{Choice of assay method}

Before the development of NGS, tumor genotyping was performed only on specific genomic loci that were known to be frequently mutated in cancer, which are known as "hotspots". These approaches were best suited to recurrent activating mutations in oncogenes, such as in the KRAS gene in colon [8] and lung cancer [9]. However, these approaches were insufficient to identify alterations in tumor suppressors (in which an alteration anywhere in the gene may impact its function) or the increasingly complex area of "long tail" hotspot alterations in oncogenes [10]. Thus, current assay options involve approaches that may capture known cancer genes ("gene panels"), whole-exome, whole-genome and/or whole-transcriptome approaches. There are several trade-offs to increasing the portion of the genome that is sequenced. The first is a loss of coverage for the same amount of sequencing (Fig. 2). Coverage, or depth, is defined as the average number of mappable reads at a given locus in your panel. Lower coverage limits the ability to confidently call a variant of low allele fraction to be biologically real and not a technical artifact. A second is that whole-genome and whole-exome sequencing require germline sequencing
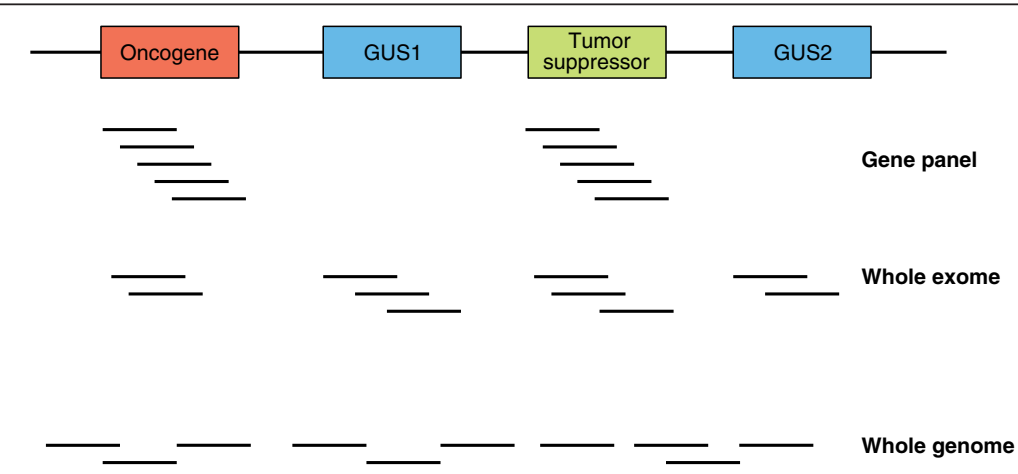

Fig. 2 The trade-off between coverage and amount of the genome covered. A hypothetical region of the genome contains an oncogene, a tumor suppressor and two genes of uncertain significance (GUS). For visual simplicity, we show ten reads, which will get sequencing depth at genes of interest. Whole-exome sequencing is able to cover each gene with fewer reads, whereas whole-genome sequencing rarely covers a specific base with more than one read. Bear in mind, this figure is vastly understating the relative size of intergenic regions. Realistic sequencing depth goals should be much higher 
to enhance the identification of true somatic variants [11], which may uncover incidental clinically relevant inherited disorders (see below).

When considering a gene panel, another decision is whether the technology should be based on hybrid capture or amplicon sequencing (Fig. 3). Amplicon sequencing enriches target genes by PCR with a set of primers for exons of selected genes prior to NGS analysis [12]. These protocols have the advantage of less required input DNA and less turnaround time than hybrid capture methods, which is critical for clinical application, but potentially PCR amplification can bias the observed allele fraction. It also pulls information out of a lower percentage of starting material, further increasing the chance of bias in calling copy number variations. The informatics analysis is relatively easy, as any read that does not map to a locus between primers can be disregarded. A downside of this simplicity is that the assay is inherently unable to detect unexpected fusions, because either the $5^{\prime}$ or $3^{\prime}$ primer would fail to bind the translocated DNA.

In hybrid capture, relevant DNA sequences are hybridized to probes, which are biotinylated. The biotin is bound to streptavidin beads and then non-bound DNA is washed away [13]. This has the advantage of more reliable detection of copy number changes, although some research groups are using amplicon-based sequencing to detect copy number changes as well [14]. The disadvantages of hybrid capture include a higher required depth of sequencing and a more advanced bioinformatics platform (see below). Hybrid capture does have the ability to detect fusion proteins, as they will be pulled down with the baited DNA. Fusions are still a challenge for hybrid capture, however, because while the fusion protein may be common, the breakpoint itself is found over the full range of an intron [15]. If there is a high suspicion that a sample may contain clinically important fusions, an assay based on cDNA should be considered. These assays will show the fused exon-exon junctions, obviating the need to find the genomic breakpoint [16]. Calling variants and DNA copy number changes can be difficult with both methods (as well as with microarray-based assays) when there is high tumor heterogeneity [17] or low tumor purity [18]. For example, a high copy number gain in a small number of cells may be interpreted as a widespread low copy number gain. Thus, putatively actionable copy number variations are typically validated by fluorescent in situ hybridization in clinical settings.

\section{Choice of clinical sample}

Most specimens that are examined by anatomical pathologists are fixed in formalin (4\% formaldehyde) and embedded in paraffin (FFPE). The formalin introduces crosslinks that can both fragment DNA and cause chemical alterations that may alter sequencing results [19]. Early studies demonstrated that using FFPE specimens in PCR-based sequencing led to more errors than using frozen specimens [20]. Some projects, including The Cancer Genome Atlas (TCGA), required the use of fresh frozen tissue [21]. There has been great progress in altering DNA extraction methods such that FFPE specimens are just as useful for NGS as fresh frozen samples [22]. While there have been some early attempts at using FFPE specimens for other modalities besides DNA

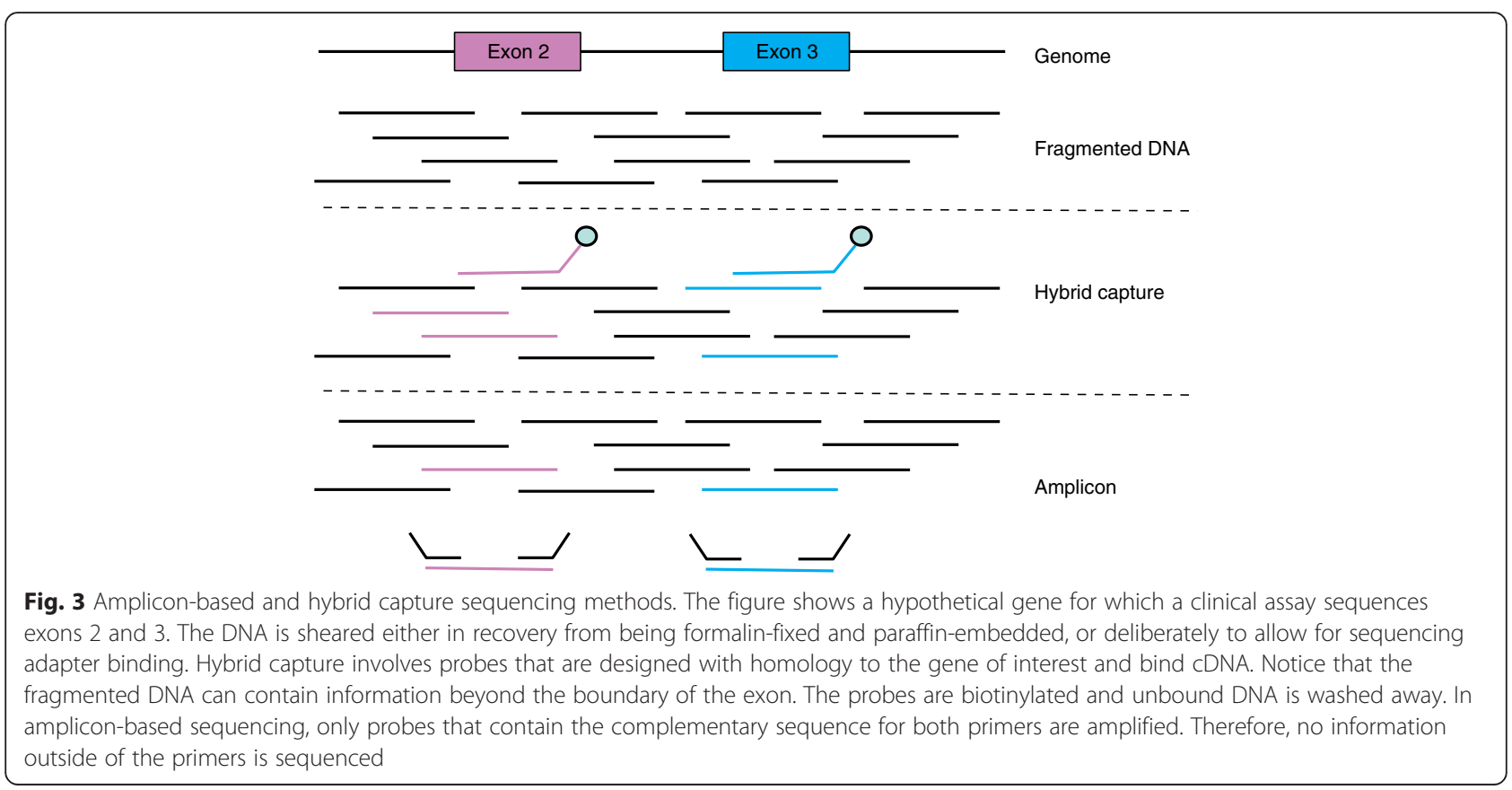


sequencing [23, 24], these tests are not yet widely used clinically, and the reliability of FFPE versus frozen samples is less well established. Clinicians should feel comfortable requesting NGS on FFPE samples, and do not necessarily have to handle the specimens differently from other diagnostic samples.

For most cancers, the standard pathological diagnosis will require a direct sample of tissue for biopsy. However, many research groups are exploring the diagnostic and therapeutic utility of "liquid biopsies". One such source of genetic material for disease monitoring are circulating tumor cells (CTCs). These suffer from a low frequency (approximately 1 cell in $10^{6}-10^{8}$ total circulating cells) and must, therefore, go through an enrichment step. A large number of CTC collection and sequencing protocols have been reported and are being evaluated prospectively [25, 26]. Alternatively, DNA released from apoptotic cells in the tumor can be assayed from the peripheral blood, and is usually referred to as circulating tumor DNA (ctDNA). Progress in utilizing ctDNA was recently reviewed [27], with the authors concluding that this approach shows great promise for the purpose of detecting minimal residual disease [28], or helping to improve diagnosis by looking for mutations specifically associated with a particular disease type [29]. RNA is much less stable than DNA in circulating blood, but RNA species can be preserved in extracellular vesicles and information about tumor recurrence can be gleaned from them as well [30]. However, reproducibility has plagued RNA-based studies, and RNA assays are not yet ready for clinical use [31].

Tumor heterogeneity is both a challenge for liquid biopsies and the reason they can be more useful than tissue biopsies [32]. Initially, mutations with a low allele fraction owing to only being present in a subset of tumor cells may be missed by liquid biopsies, as the low amount of DNA input to the assay is compounded by the low incidence of the mutation. This makes distinguishing low allele fraction mutants from errors that are inherent to high-throughput sequencing very difficult (see below). However, the ability for minimally invasive samples to be sequenced repeatedly over time will allow for faster recognition of known resistance mutations. Sequencing artifacts should be random, but sequences that appear serially can be weighted and followed more closely. It should also be noted that errors in aligning reads to the correct locus will give what appear to be recurrent mutations, so all mutations that are used for serial tracking of tumor burden should be manually reviewed. Overall, there is much promise in sequencing tumor DNA from peripheral blood, but its use is still under investigation and clinicians should rely on other methods for tracking disease progression.

\section{Clinical NGS data analysis}

An additional area of innovation for clinical NGS involves bioinformatic analysis of raw genomic data and rapid clinical interpretation for consideration by the treating clinician. The first step in this process is to assign a genetic location to the read by mapping it on a reference genome [3]. Some percentage of the reads will be "unmappable", that is, the software cannot assign the sequence to a unique genomic location [33]. An individual genome will have a number of deviations from a reference genome, referred to as single nucleotide variants (SNVs), and/or structural alterations such as insertions, deletions or translocations. Somatic mutation analysis, as is done in cancer, involves a number of additional challenges. There are robust algorithms available for identifying many clinically relevant alterations that occur as point mutations, short insertions or deletions, or copy number aberrations in clinical samples analyzed by NGS [34].

However, as DNA mutations accumulate within a tumor, there can be considerable sequence heterogeneity even within a single primary tumor [17]. It can be very challenging to discern whether a read of a low allele fraction represents a true mutation that exists within a subset of tumor cells or is an artifact that should be discarded. While retrospective research endeavors may not require the identification of all possible clinically actionable alterations in a cohort study, prospective clinical cancer genomics requires increased sensitivity to detect low allelic fraction alterations in impure tumor samples that may impact an individual patient's care. These issues can be exacerbated by low amount of tumor relative to normal tissue within the sample and mitigated by having more reads, that is, greater coverage. If a detected mutation is the result of a low allele fraction within the sample, the number of reads will rise proportionally with total reads, whereas if it is a technical artifact, the number of reads should be random and can be eliminated from analysis. Estimating tumor percentage from a standard pathology specimen should be helpful for giving an expected allele fraction within the sample, but is prone to very high inter-observer variation [35].

A second challenge is frequent DNA fusions, which represent a significant component of the clinically actionable spectrum of alterations in oncology (for example, $A L K$ fusions, $B C R-A B L$ fusions). Within NGS data, these events will cause both ends of a read to be mappable, but the whole contiguous sequence is not. This is referred to as a split read, and can be challenging in the presence of a high number of structural rearrangements, such as in cancers with chromothripsis [36]. Notably, since most clinically relevant somatic fusions occur outside of coding regions, whole-exome sequencing assays often miss these variants, and gene 
panels that are not designed to cover known fusion territories will also be unable to identify these fusion products. Thus, when analyzing a clinical NGS data set, it is critical to understand the analytical limitations of a given assay as represented in the downstream data analysis.

\section{Clinical interpretation of NGS data}

After identification of the set of alterations within a given patient's tumor, many cases will yield a small set of clinically relevant events as well as a long list of sequencing variants of uncertain significance. An emerging body of interpretation algorithms that automate the clinical relevance of the alterations will enable more rapid clinical interpretation of cancer genomic sequencing data. For instance, one algorithm called PHIAL applies a heuristic method to rank alterations by clinical and biological relevance, followed by intra-sample pathway analysis to determine potentially druggable nodes [22, 37]. As such approaches mature, they will be better equipped to apply tumor-specific "priors" to the genomic data, along with genotype-phenotype therapeutic outcomes data, to enable probabilistic approaches to ranking tumor genomic alterations by clinical relevance.

Furthermore, there are several databases that can be accessed to evaluate the clinical significance of mutations. The first level of analysis is whether the variant you are interested in has been seen before in published reports. A simple concept is that driver mutations are more likely to recur across multiple patients and tumor types. The most common databases used (Table 1) are the Catalog of Somatic Mutations in Man (COSMIC) [38, 39], and TCGA (available for data exploration at multiple sites) [40, 41]. After whittling the mutations down to those that are recurrent, information about therapies and prognostic information can be found at a number of locations. Cancer centers that have created and host these databases include MD Anderson's Personalized Cancer Therapy [42, 43], Vanderbilt's My Cancer Genome [44, 45], and the Broad Institute's TARGET $[22,46]$. Each database contains useful information and links to relevant primary literature. Moving forward, there will have to be more steps to improve data sharing, with the creation of a central repository of both sequences and de-identified patient information, but there is no consensus yet for how this process should happen.

Finally, for NGS technologies that require both somatic and germline testing (for example, whole-exome and whole-genome sequencing), the American College of Medical Genetics has released guidelines outlining which variants should always be reported to patients regardless of whether they are relevant to the presenting illness [47]. Since most of these genes involve noncancer-related syndromes, there is an increasing need for oncologists to be prepared to receive results that bring up unexpected inherited genetic issues [48]. However, the germline component to clinical oncology NGS testing may have significant diagnostic and therapeutic utility, as demonstrated by the identification of pathogenic germline alterations in men with castration-resistant prostate cancer who respond to PARP inhibition [49], and its role in this arena is evolving rapidly.

\section{NGS utility}

There are three general ways that NGS can aid a clinician. The first is with diagnosis; tumor subtypes that only a few years ago were defined by morphologic criteria are now defined by genetic mutations, either inclusively or exclusively. For example, $15 / 15$ patients in a study looking at fibrolamellar hepatocellular carcinoma had an in-frame fusion between DNAJB1 and PRKACA [50]. The second is finding an appropriate "targeted therapy", as an increasing number of therapies have indications based on DNA sequencing results (Table 2). Patients who lack the mutation targeted by a drug will not only fail to benefit, but can actually be harmed by inappropriate targeted therapies [51]. The third point at which clinicians stand to benefit from NGS is when a patient stops responding to a targeted therapy with known resistance mutations. In some instances, the resistance mutation may be limited to one or a few loci. For example, resistance to EGFR targeted therapies in cancer very frequently involves a single point mutation,

Table 1 Recommended databases for interpreting somatic mutation results in cancer

\begin{tabular}{llll}
\hline Database & Institute & Organized by & Reference \\
\hline TARGET & BROAD & Gene & {$[46]$} \\
PCT & MD Anderson & Gene & {$[43]$} \\
CBioPortal & MSK & TCGA diseases & {$[41]$} \\
COSMIC & Sanger & Gene & {$[39]$} \\
IntOGen & University Pompeu Fabra & Gene & {$[73]$} \\
My Cancer Genome & Vanderbilt & Disease & {$[45]$} \\
CIViC & Washington University & Variant & [74] \\
DGldb & Washington University & Drug/gene interaction & [75] \\
\hline
\end{tabular}

Each database is listed with hosting institution, website, and the primary search term by which it is organized. TCGA The Cancer Genome Atlas 
Table 2 FDA-approved drugs with a companion diagnostic

\begin{tabular}{|c|c|c|c|}
\hline Drug & Disease & DNA mutation & Action \\
\hline Imatinib, Dasatinib, Nilotinib, Bosutinib & Chronic myelogenous leukemia & $B C R-A B L 1$ fusion & Indication for therapy \\
\hline \multirow[t]{2}{*}{ Ponatinib } & \multirow[t]{2}{*}{ Chronic myelogenous leukemia } & $B C R-A B L 1$ fusion & \multirow[t]{2}{*}{ Only indicated for $\mathrm{T} 315 \mathrm{I}$ mutations } \\
\hline & & T315I resistance mutation & \\
\hline \multirow[t]{3}{*}{ Erlotinib, Afatinib } & \multirow[t]{3}{*}{ Lung adenocarcinoma } & EGFR & \multirow[t]{3}{*}{ Indication for therapy } \\
\hline & & Exon 19 deletions & \\
\hline & & L858R & \\
\hline Vemurafenib, Dabrafenib & Melanoma & BRAF V600E & Indication for therapy \\
\hline Tramatenib & Melanoma & BRAF V600E/K & Indication for therapy \\
\hline Crizotinib & Lung cancer & ALK gene fusions & Indication for therapy \\
\hline Cetuximab & Colon cancer & KRAS codon 12, 13 & Contraindication to therapy \\
\hline Olaparib & Ovarian cancer & $B R C A 1$ and $B R C A 2$ mutations & Indication for therapy \\
\hline
\end{tabular}

Each drug has a specific genomic result that is part of its indication for use. FDA Food and Drug Administration

and can possibly be overcome by merely switching to a different agent [52]. However, glioblastoma can become resistant to EGFR targeted therapies via a complicated epigenetic regulation [53]. NGS allows a more complete overview of tumor dynamics, and is more likely to shed light on idiopathic resistance mechanisms than a single gene assay.

If a patient has failed conventional therapy, NGS can be immensely helpful for identifying and enrolling them into an appropriate clinical trial. There are two types of clinical trial structure that require patients to have their tumors' genetic makeup well defined by NGS (Fig. 4). In an umbrella trial, patients with a type of morphologically defined cancer are assigned to a treatment arm on the basis of the genetic mutations detected in their tumor. Umbrella trials comprise many different treatment arms under the umbrella of a single trial. In essence, umbrella trials test whether a "precision" approach leads to better outcomes within a traditional diagnosis (for example, lung adenocarcinoma) than standard of care approaches. In a bucket trial (also called a basket trial), cancers of different types are clustered exclusively by genetic mutation. The US National Cancer Institute has recognized the potential of the NGS followed by targeted therapy

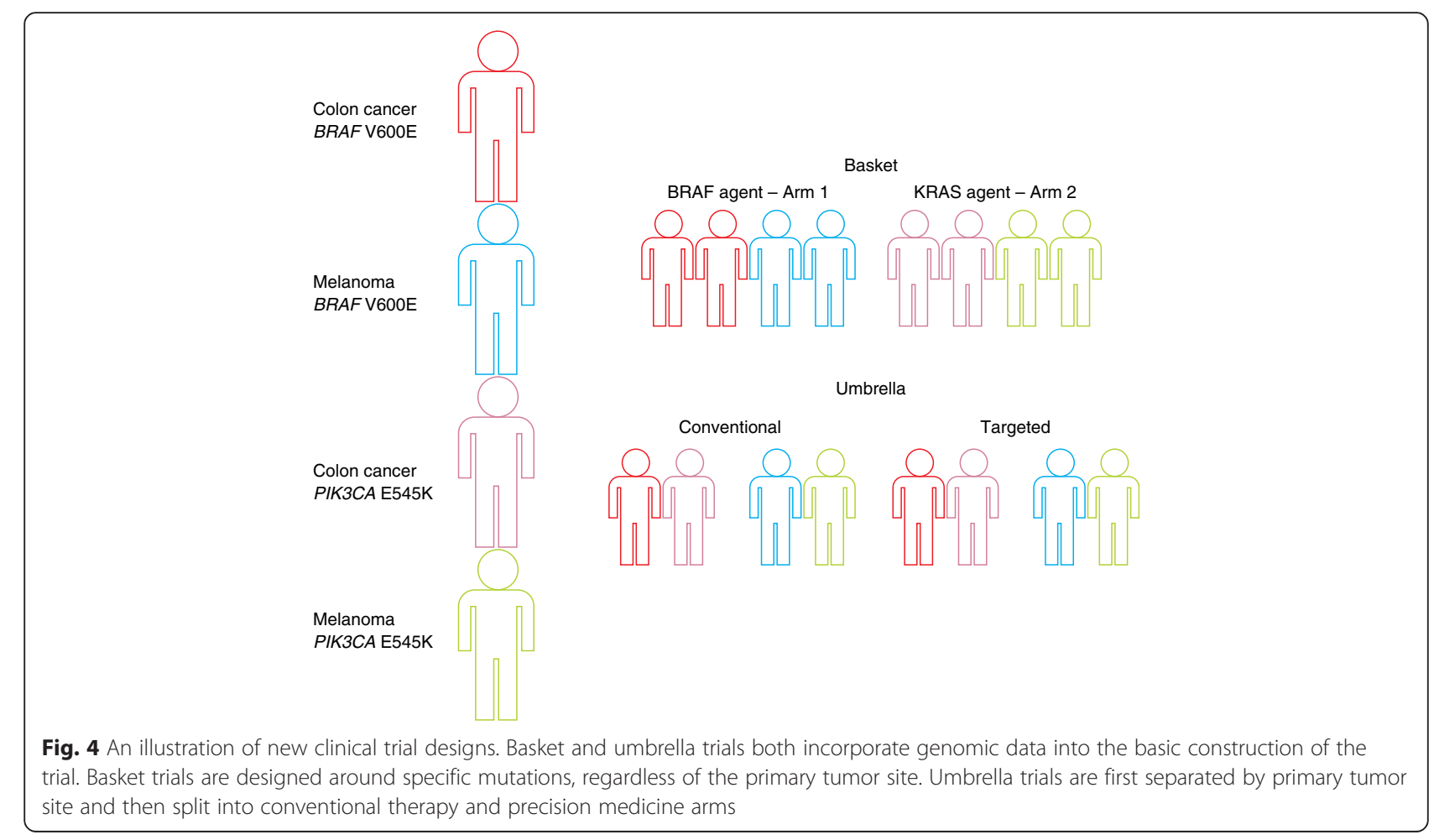


approach by setting up the Molecular Analysis for Therapy Choice (MATCH) Program. Biopsies from tumors from as many as 3000 patients will undergo NGS to identify individuals whose tumors have genetic abnormalities that may respond to selected targeted drugs. As many as 1000 patients will then be assigned to one of the phase II trials, with assignment based not on their type of cancer but on the genetic abnormality that is thought to be driving their cancer [54]. The nuances of constructing these types of trials are beyond the scope of this review and have been covered well previously [55].

Efforts are ongoing to determine prognostic biomarkers in clinical oncology. Many false starts have been caused by extrapolating from what is called overfitting, which is building a precise model from a small, nonrepresentative data set. Determining prognosis on the basis of non-druggable mutations from NGS has tended to follow from this tradition. Certain mutations, such as TP53 [56], portend a poor prognosis in almost all clinical situations. Others, such as ASXL1, are only associated with a particular disease [57]. Mutations in IDH1 and $I D H 2$ indicate a better prognosis in glioma [58], but often show contradictory results in myeloid malignancies
[59], although this may change as targeted agents move through clinical trials [60]. Caution should be used when communicating prognostic information to patients.

\section{Clinical NGS case study}

As an example that demonstrates the utility of clinical NGS, we look at the fictional scenario of a patient who presents with a newly diagnosed lung adenocarcinoma (Fig. 5). Targeted therapies that affect multiple recurrent alterations in lung adenocarcinoma have been developed, including those that target EGFR mutations, MET amplification, and $A L K$ or ROS1 fusions, among others [61]. Thus, a targeted gene panel that encompasses these events would be most commonly applied. DNA can be harvested from the FFPE tumor block obtained from a diagnostic biopsy sample, and targeted NGS sequencing can be used to identify the set of somatic point mutations, short insertions/deletions, copy number alterations, and oncogenic fusion events. In this case, let us say that the resulting inter pretation of the set of variants reveals two mutations: EGFR L858R (allelic fraction of $35 \%$ ) and TP53 R273H (allelic fraction of $80 \%$ ). All databases

Choose approach

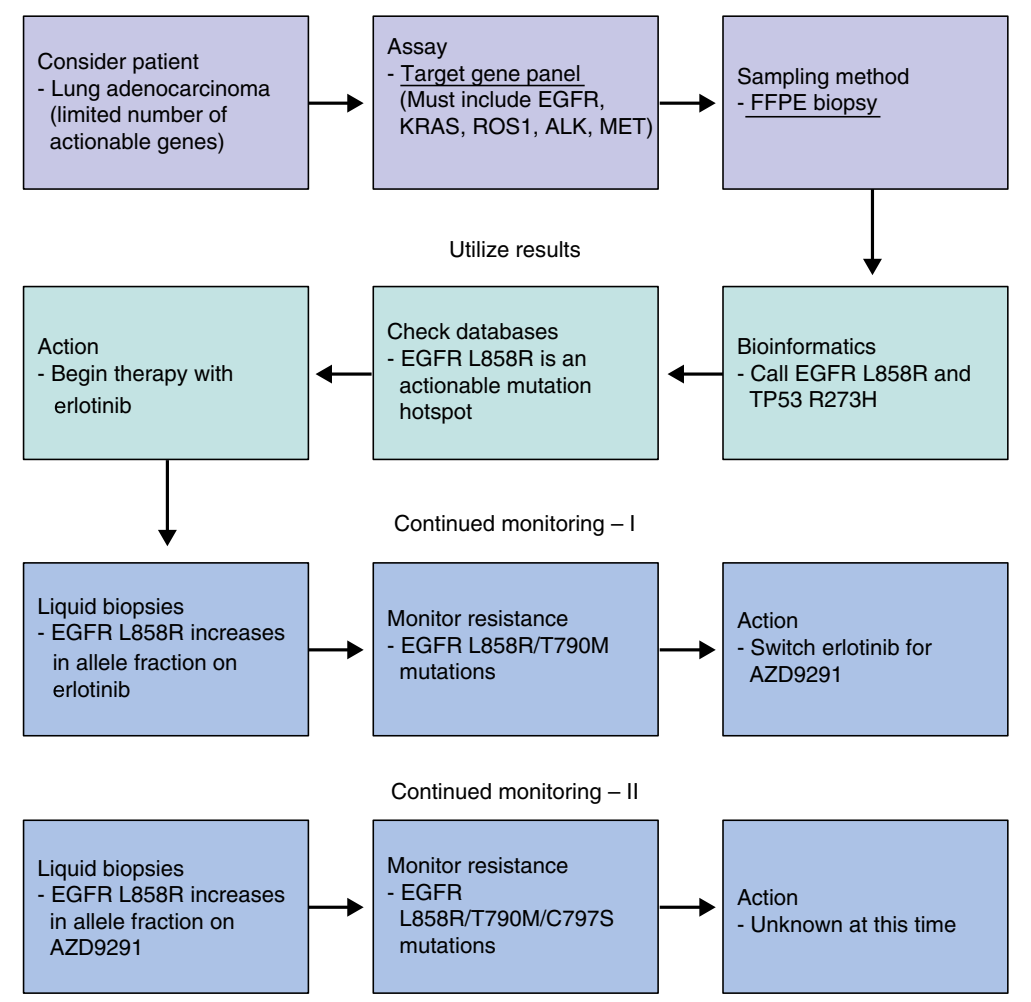

Fig. 5 A representative clinical example of how NGS is utilized in recurrent lung adenocarcinoma. The illustrative case from the text has been fitted to the outline in Fig. 1. In a lung adenocarcinoma, there are a number of actionable mutations; this case shows a canonical EGFR mutation, treated with erlotinib. There are actually now two levels of resistance that can develop, illustrated in rows 3 and 4 . FFPE formalin-fixed, 
highlight that EGFR L858R mutations are sensitizing for erlotinib. The TP53 mutation likely confers a worse prognosis [62], but management does not change as a result. The patient can be followed by both radiology and/or ctDNA assays, with the L858R mutation as a marker of tumor DNA [29]. The patient has a good initial response but develops a recurrence after 6 months. Repeat biopsy and NGS testing is obtained, which reveals the L858R mutation with a $35 \%$ allele fraction and a second EGFR T790M mutation with a $12 \%$ allele fraction. From this it would be possible to infer that the second mutation in $E G F R$ is derived from a resistant subclone that has emerged as a result of therapy, as indicated by the lower allelic fraction compared with the original EGFR mutation. The databases show that this is a common resistance mutation for erlotinib, but can be targeted by newer agents [52, 63]. The patient should continue to be followed, because these newer agents can also trigger the development of additional resistance mutations [52, 64, 65] in EGFR or other genes (thereby highlighting the need for broader testing using NGS beyond limited gene testing to ensure identification of the resistance alteration).

\section{Future directions}

While much information can be gleaned from a tumor DNA sequence, we must be mindful that DNA itself is rather inert. Better information about the functionality of a cancer can be obtained by integrating information from different modalities. RNA sequencing could give information about the relative expression of a mutated gene. Approaches in mass spectrometry are giving a clearer picture of the proteomics of cancer [66]. TCGA data were collected using a number of different modalities, and are available for several tumor types, and while useful information can be gleaned at different levels, tying everything together remains a prodigious challenge [67]. The methods used to predict phenotypes from integrated -omics data have been reviewed recently [68].

Furthermore, immunotherapies are quickly gaining prevalence for cancer therapy, especially for use in melanoma [69]. NGS sequencing could become very important for predicting responses to immunotherapy. Neoantigens - that is, antigens that are created by somatic mutations - are correlated with the overall rate of somatic mutation and clinical response [70]. Immune response is mediated by T-cell recognition of these neoantigens [71]. Exome sequencing can be paired with mass spectrometry to determine which neoantigens are successfully presented by the major histocompatibility complex (MHC) [72].

\section{Conclusion}

NGS is inextricably intertwined with the realization of precision medicine in oncology. While it is unlikely to obviate traditional pathologic diagnosis in its current state, it allows a more complete picture of cancer etiology than can be seen with any other modality. However, precision cancer medicine and large-scale NGS testing will require novel approaches towards ensuring evidence-based medicine. Treating each genetic abnormality as an independent variable when hundreds or thousands are queried in every patient will require new trial designs and statistical methods to ensure the utility of these approaches. Broadly, clinicians and translational researchers will need to continue to engage in direct dia$\log$, both within and across institutions, to advance the integration of genomic information and clinical phenotypes, and enable precision cancer medicine through NGS approaches.

\section{Abbreviations \\ CTC: Circulating tumor cell; ctDNA: circulating tumor DNA; FDA: Food and Drug Administration; FFPE: Formalin-fixed, paraffin-embedded; MATCH: Molecular Analysis for Therapy Choice; MHC: Major histocompatibility complex; NGS: Next-generation sequencing; SNV: Single nucleotide variant; TCGA: The Cancer Genome Atlas.}

\section{Competing interests}

EMVA has a consultant or advisory role with Syapse and Roche Ventana.

\section{Acknowledgements}

This work is supported by the National Institutes of Health 1K08CA188615 (EMVA), Prostate Cancer Foundation (EMVA), and the American Cancer Society (EMVA).

\section{Author details}

${ }^{1}$ Department of Pathology, Brigham and Women's Hospital, Boston, MA 02115, USA. ${ }^{2}$ Department of Medical Oncology, Dana-Farber Cancer Institute, Boston, MA 02115, USA. ${ }^{3}$ Broad Institute of MIT and Harvard, Cambridge, MA 02142, USA.

Published online: 29 July 2015

\section{References}

1. Van Regenmortel MH. Reductionism and complexity in molecular biology. Scientists now have the tools to unravel biological and overcome the limitations of reductionism. EMBO Rep. 2004;5:1016-20.

2. Gatherer D. So what do we really mean when we say that systems biology is holistic? BMC Syst Biol. 2010;4:22.

3. Meyerson M, Gabriel S, Getz G. Advances in understanding cancer genomes through second-generation sequencing. Nat Rev Genet. 2010;11:685-96.

4. Ledford $\mathrm{H}$. Big science: the cancer genome challenge. Nature. 2010;464:972-4.

5. Reuter JA, Spacek DV, Snyder MP. High-throughput sequencing technologies. Mol Cell. 2015;58:586-97.

6. Roychowdhury S, lyer MK, Robinson DR, Lonigro RJ, Wu YM, Cao X, et al. Personalized oncology through integrative high-throughput sequencing: a pilot study. Sci Transl Med. 2011;3:111ra121.

7. Garraway LA. Genomics-driven oncology: framework for an emerging paradigm. J Clin Oncol. 2013;31:1806-14.

8. De Roock W, De Vriendt V, Normanno N, Ciardiello F, Tejpar SKRAS, BRAF. PIK3CA, and PTEN mutations: implications for targeted therapies in metastatic colorectal cancer. Lancet Oncol. 2011;12:594-603.

9. Meng D, Yuan M, Li Z, Chen L, Yang J, Zhao X, et al. Prognostic value of K-RAS mutations in patients with non-small cell lung cancer: a systematic review with meta-analysis. Lung Cancer. 2013;81:1-10. 
10. Garraway LA, Lander ES. Lessons from the cancer genome. Cell. 2013;153:17-37.

11. Jones S, Anagnostou V, Lytle K, Parpart-Li S, Nesselbush M, Riley DR, et al. Personalized genomic analyses for cancer mutation discovery and interpretation. Sci Transl Med. 2015;7:283ra253.

12. Chang F, Li MM. Clinical application of amplicon-based next-generation sequencing in cancer. Cancer Genet. 2013;206:413-19.

13. Wagle N, Berger MF, Davis MJ, Blumenstiel B, DeFelice M, Pochanard P, et al. High-throughput detection of actionable genomic alterations in clinical tumor samples by targeted, massively parallel sequencing. Cancer Discov. 2012;2:82-93.

14. Grasso C, Butler T, Rhodes K, Quist M, Neff TL, Moore S, et al. Assessing copy number alterations in targeted, amplicon-based next-generation sequencing data. J Mol Diagn. 2015;17:53-63.

15. Rabbitts TH. Commonality but diversity in cancer gene fusions. Cell. 2009;137:391-5.

16. Suehara $Y$, Arcila $M$, Wang $L$, Hasanovic A, Ang D, Ito T, et al. Identification of KIF5B-RET and GOPC-ROS1 fusions in lung adenocarcinomas through a comprehensive mRNA-based screen for tyrosine kinase fusions. Clin Cancer Res. 2012;18:6599-608.

17. Gerlinger M, Rowan AJ, Horswell S, Larkin J, Endesfelder D, Gronroos E, et al. Intratumor heterogeneity and branched evolution revealed by multiregion sequencing. N Engl J Med. 2012;366:883-92.

18. Gusnanto A, Wood HM, Pawitan Y, Rabbitts P, Berri S. Correcting for cancer genome size and tumour cell content enables better estimation of copy number alterations from next-generation sequence data. Bioinformatics. 2012;28:40-7.

19. Do H, Dobrovic A. Sequence artifacts in DNA from formalin-fixed tissues: causes and strategies for minimization. Clin Chem. 2015;61:64-71.

20. Williams $C$, Pontén F, Moberg C, Söderkvist $P$, Uhlén M, Pontén J, et al. A high frequency of sequence alterations is due to formalin fixation of archival specimens. Am J Pathol. 1999:155:1467-71.

21. Goetz L, Bethel K, Topol EJ. Rebooting cancer tissue handling in the sequencing era: toward routine use of frozen tumor tissue. JAMA. 2013;309:37-8

22. Van Allen EM, Wagle $N$, Stojanov P, Perrin DL, Cibulskis K, Marlow S, et al. Whole-exome sequencing and clinical interpretation of formalin-fixed, paraffin-embedded tumor samples to guide precision cancer medicine. Nat Med. 2014;20:682-8.

23. Hedegaard J, Thorsen K, Lund MK, Hein A-M K, Hamilton-Dutoit SJ, Vang S, et al. Next-generation sequencing of RNA and DNA isolated from paired fresh-frozen and formalin-fixed paraffin-embedded samples of human cancer and normal tissue. PLoS One. 2014:9, e98187.

24. Dumenil TD, Wockner LF, Bettington M, McKeone DM, Klein K, Bowdler LM, et al. Genome-wide DNA methylation analysis of formalin-fixed paraffin embedded colorectal cancer tissue. Genes Chromosomes Cancer. 2014:53:537-48.

25. Joosse SA, Gorges TM, Pantel K. Biology, detection, and clinical implication of circulating tumor cells. EMBO Mol Med. 2015;7:1-11.

26. Lohr JG, Adalsteinsson VA, Cibulskis K, Choudhury AD, Rosenberg M, Cruz-Gordillo $P$, et al. Whole-exome sequencing of circulating tumor cells provides a window into metastatic prostate cancer. Nat Biotechnol. 2014:32:479-84.

27. Diaz LA, Bardelli A. Liquid biopsies: genotyping circulating tumor DNA. J Clin Oncol. 2014:32:579-86.

28. Dawson SJ, Tsui DWY, Murtaza M, Biggs H, Rueda OM, Chin S-F, et al. Analysis of circulating tumor DNA to monitor metastatic breast cancer. N Engl J Med. 2013;368:1199-209.

29. Newman AM, Bratman SV, To J, Wynne JF, Eclov NCW, Modlin LA, et al. An ultrasensitive method for quantitating circulating tumor DNA with broad patient coverage. Nat Med. 2014;20:548-54.

30. Redzic JS, Balaj L, van der Vos KE, Breakefield XO. Extracellular RNA mediates and marks cancer progression. Semin Cancer Biol. 2014;28:14-23.

31. SEQC/MAQC-III Consortium. A comprehensive assessment of RNA-seq accuracy, reproducibility and information content by the Sequencing Quality Control Consortium. Nat Biotechnol. 2014:32:903-14.

32. Janku F. Tumor heterogeneity in the clinic: is it a real problem? Ther Adv Med Oncol. 2014:6:43-51.

33. Li W, Freudenberg J. Characterizing regions in the human genome unmappable by next-generation-sequencing at the read length of 1000 bases. Comput Biol Chem. 2014;53:108-17.
34. Van Allen EM, Wagle N, Levy MA. Clinical analysis and interpretation of cancer genome data. J Clin Oncol. 2013;31:1825-33.

35. Smits AJJ, Kummer JA, de Bruin PC, Bol M, van den Tweel JG, Seldenrijk KA, et al. The estimation of tumor cell percentage for molecular testing by pathologists is not accurate. Mod Pathol. 2014;27:168-74.

36. Forment JV, Kaidi A, Jackson SP. Chromothripsis and cancer: causes and consequences of chromosome shattering. Nat Rev Cancer. 2012;12:663-70

37. PHIAL. http://www.broadinstitute.org/cancer/cga/phial.

38. Forbes SA, Tang G, Bindal N, Bamford S, Dawson E, Cole C, et al. COSMIC (the Catalogue of Somatic Mutations in Cancer): a resource to investigate acquired mutations in human cancer. Nucleic Acids Res. 2010;38:D652-7.

39. COSMIC. http://cancer.sanger.ac.uk/cosmic/.

40. Cerami E, Gao J, Dogrusoz U, Gross BE, Sumer SO, Aksoy BA, et al. The cBio cancer genomics portal: an open platform for exploring multidimensional cancer genomics data. Cancer Discov. 2012;2:401-4.

41. cBioPortal. http://www.cbioportal.org/.

42. Meric-Bernstam F, Johnson A, Holla V, Bailey AM, Brusco L, Chen K, et al. A decision support framework for genomically informed investigational cancer therapy. J Natl Cancer Inst. 2015;107:djv098. doi:10.1093/jnci/djv098.

43. Personalized Cancer Therapy. https://pct.mdanderson.org/.

44. Yeh $\mathrm{P}, \mathrm{Chen} \mathrm{H}$, Andrews J, Naser R, Pao W, Horn L. DNA-mutation inventory to refine and enhance cancer treatment (DIRECT): a catalog of clinically relevant cancer mutations to enable genome-directed anticancer therapy. Clin Cancer Res. 2013;19:1894-901.

45. My Cancer Genome. http://www.mycancergenome.org/.

46. TARGET. https://www.broadinstitute.org/cancer/cga/target.

47. Green RC, Berg JS, Grody WW, Kalia SS, Korf BR, Martin CL, et al. ACMG recommendations for reporting of incidental findings in clinical exome and genome sequencing. Genet Med. 2013;15:565-74.

48. Parsons DW, Roy A, Plon SE, Roychowdhury S, Chinnaiyan AM. Clinical tumor sequencing: an incidental casualty of the American College of Medical Genetics and Genomics recommendations for reporting of incidental findings. J Clin Oncol. 2014;32:2203-5.

49. Robinson D, Van Allen EM, Wu Y-M, Schultz N, Lonigro RJ, Mosquera J$\mathrm{M}$, et al. Integrative clinical genomics of advanced prostate cancer. Cell. 2015;161:1215-28.

50. Honeyman JN, Simon EM, Robine N, Chiaroni-Clarke R, Darcy DG, Lim IIP, et al. Detection of a recurrent DNAJB1-PRKACA chimeric transcript in fibrolamellar hepatocellular carcinoma. Science. 2014;343:1010-14.

51. Douillard JY, Oliner KS, Siena S, Tabernero J, Burkes R, Barugel M, et al. Panitumumab-FOLFOX4 treatment and RAS mutations in colorectal cancer. N Engl J Med. 2013;369:1023-34.

52. Jänne PA, Yang JC-H, Kim D-W, Planchard D, Ohe Y, Ramalingam SS, et al. AZD9291 in EGFR inhibitor-resistant non-small-cell lung cancer. N Engl J Med. 2015;372:1689-99.

53. Nathanson DA, Gini B, Mottahedeh J, Visnyei K, Koga T, Gomez G, et al. Targeted therapy resistance mediated by dynamic regulation of extrachromosomal mutant EGFR DNA. Science. 2014:343:72-6.

54. Abrams J, Conley B, Mooney M, Zwiebel J, Chen A, Welch JJ, et al. National Cancer Institute's Precision Medicine Initiatives for the new National Clinical Trials Network. Am Soc Clin Oncol Educ Book. 2014;34:71-6.

55. Redig AJ, Jänne PA. Basket trials and the evolution of clinical trial design in an era of genomic medicine. J Clin Oncol. 2015;33:975-7.

56. Vousden $\mathrm{KH}$, Prives C. P53 and prognosis: new insights and further complexity. Cell. 2005;120:7-10.

57. Gelsi-Boyer V, Brecqueville M, Devillier R, Murati A, Mozziconacci M-J, Birnbaum D. Mutations in ASXL1 are associated with poor prognosis across the spectrum of malignant myeloid diseases. J Hematol Oncol. 2012;5:12.

58. Yan H, Williams Parsons D, Jin G, McLendon R, Ahmed Rasheed B, Yuan W, et al. IDH1 and IDH2 mutations in gliomas. N Engl J Med. 2009;360:765-73.

59. Im AP, Sehgal AR, Carroll MP, Smith BD, Tefferi A, Johnson DE, et al. DNMT3A and IDH mutations in acute myeloid leukemia and other myeloid malignancies: associations with prognosis and potential treatment strategies. Leukemia. 2014;28:1774-83.

60. Wang F, Travins J, DeLaBarre B, Penard-Lacronique V, Schalm S, Hansen E, et al. Targeted inhibition of mutant IDH2 in leukemia cells induces cellular differentiation. Science. 2013;340:622-6.

61. Camidge DR, Pao W, Sequist LV. Acquired resistance to TKls in solid tumours: learning from lung cancer. Nat Rev Clin Oncol. 2014;11:473-81. 
62. Ahrendt SA, Hu Y, Buta M, McDermott MP, Benoit N, Yang SC, et al. p53 mutations and survival in stage I non-small-cell lung cancer: results of a prospective study. J Natl Cancer Inst. 2003;95:961-70.

63. Sequist LV, Soria J-C, Goldman JW, Wakelee HA, Gadgeel SM, Varga A, et al. Rociletinib in EGFR-mutated non-small-cell lung cancer. N Engl J Med. 2015:372:1700-9.

64. Thress KS, Paweletz CP, Felip E, Cho BC, Stetson D, Dougherty B, et al. Acquired EGFR C797S mutation mediates resistance to AZD9291 in nonsmall cell lung cancer harboring EGFR T790M. Nat Med. 2015;21:560-2.

65. Niederst MJ, Hu H, Mulvey HE, Lockerman EL, Garcia AR, Piotrowska Z, et al. The allelic context of the C797S mutation acquired upon treatment with third generation EGFR inhibitors impacts sensitivity to subsequent treatment strategies. Clin Cancer Res. 2015. doi:10.1158/1078-0432.CCR-150560 .

66. Hanash S, Taguchi A. The grand challenge to decipher the cancer proteome. Nat Rev Cancer. 2010;10:652-60.

67. Hoadley KA, Yau C, Wolf DM, Cherniack AD, Tamborero D, Ng S, et al. Multiplatform analysis of 12 cancer types reveals molecular classification within and across tissues of origin. Cell. 2014;158:929-44.

68. Ritchie MD, Holzinger ER, Li R, Pendergrass SA, Kim D. Methods of integrating data to uncover genotype-phenotype interactions. Nat Rev Genet. 2015;16:85-97.

69. Robert C, Schachter J, Long GV, Arance A, Grob JJ, Mortier L, et al. Pembrolizumab versus ipilimumab in advanced melanoma. N Engl J Med. 2015;372:2521-32.

70. Schumacher TN, Schreiber RD. Neoantigens in cancer immunotherapy. Science. 2015;348:69-74.

71. Linnemann C, van Buuren MM, Bies L, Verdegaal LME, Schotte $R$, Calis JJA, et al. High-throughput epitope discovery reveals frequent recognition of neo-antigens by CD4 ${ }^{+} T$ cells in human melanoma. Nat Med. 2015;21:81-5

72. Yadav M, Jhunjhunwala S, Phung WT, Lupardus P, Tanguay J, Bumbaca S, et al. Predicting immunogenic tumour mutations by combining mass spectrometry and exome sequencing. Nature. 2014;515:572-6.

73. IntOGen. https://www.intogen.org/.

74. CIViC. https://civic.genome.wustl.edu/\#/home.

75. DGldb. http://www.dgidb.org/. 